\title{
LncRNA IGBP1-AS1/miR-24-1/ZIC3 loop regulates the proliferation and invasion ability in breast cancer
}

\author{
Deqin Chen, Yangfan Fan and Fang Wan*
}

\begin{abstract}
Background: Breast cancer (BC) is one of the malignant solid tumors with the highest morbidity in the world. Currently, the therapeutic outcome of different types of treatment can be unsatisfactory. Novel IncRNA biomarkers in BC remains to be further explored.

Methods: Different expression of IncRNAs among BC tissues and adjacent normal tissues were identified with microarray analyses. A series of in vivo and in vitro gain-of-function laboratory procedures were conducted to study the biological functions of IGBP1-AS1. The prognostic effects on IGBP1-AS1 survival were evaluated by using in situ hybridization and survival analysis. In addition, other experiments including RNA pull down analysis, RNA immunoprecipitation, luciferase reporter assays, and chromatin immunoprecipitation as well as validating assays conducted in vivo were applied to identify the target and regulatory mechanisms of IGBP1-AS1.
\end{abstract}

Results: Significant down-regulation of IGBP1-AS1 was discovered in the cell lines and tissues of BC. With respect to its biological function, overexpression of IGBP1-AS1 had inhibitory effects on the invasion and proliferation of BC cells in vivo as well as in vitro. Analysis of the samples obtained from BC patients indicated a positive effect of IGBP1-AS1 on survival outcomes. LnCRNA IGBP1-AS1/miR-24-1/ZIC3 axis as a loop can regulate the proliferation and invasion of BC cells.

Conclusions: IGBP1-AS1 could have inhibitory impact on the invasion and proliferation of $\mathrm{BC}$ and may serve as a promising biomarker for BC.

Keywords: Breast cancer, IGBP1-AS1, miR-24-1, ZIC3

\section{Background}

The morbidity and mortality rates of breast cancer $(\mathrm{BC})$ worldwide are more than $20 \%$ and $10 \%$, respectively [1]. Currently, treatment for BC includes surgery, chemotherapy, radiotherapy, endocrine therapy and targeted therapy however, the therapeutic effect often remains unsatisfactory. Long-term continuous chemoradiotherapy can lead to toxic side effects and weaken the patient, while the cytotoxic effect on cancer cells is also poor

*Correspondence: 5504009@zju.edu.cn

Department of Surgery, The Women's Hospital, School of Medicine,

Zhejiang University, Hangzhou, Zhejiang, China
$[2,3]$. Current targeted therapies for BC mainly include targeted endocrine therapy for hormone receptor-positive $B C$ and epidermal growth factor receptor (HER)2-positive BC. However, the use of trastuzumab in the clinical treatment of early HER2-positive and metastatic BCs benefits less than $35 \%$ of patients [4], and the development of drug resistance is an additional problem that needs to be urgently solved.

Human genomics research has often focused on RNAs that encode proteins. RNAs that cannot encode proteins are considered useless RNAs or transcriptional noise. However, these RNAs play an indispensable role in important activities such as cell proliferation, 
differentiation, senescence and apoptosis. In mammalian genomics, only a small number of transcripts encode proteins, and the vast majority are noncoding RNAs (ncRNAs), accounting for approximately $80 \%$ of the human genome. According to the size of the transcript, ncRNAs can be divided into miRNAs and lncRNAs. NcRNAs greater than $200 \mathrm{nt}$ in length are called lncRNAs [5]. Ponting et al. [6] studied the evolution of lncRNAs and their role in transcriptional regulation and epigenetic gene regulation. They speculated that lncRNAs may have five different origins: generated by (1) protein-coding gene sequence disruption; (2) chromatin rearrangement; (3) noncoding RNA reverse transcriptional translocation; (4) noncoding genes containing adjacent repeats; and (5) insertion of a transposable element into the genome. However, there is still no definitive theory about the origin of lncRNAs. The biological functions of lncRNA are mainly accomplished through signaling molecules, decoy molecules, guide molecules, scaffold molecules and chromatin modification complexes, occurring at both the transcriptional level and the posttranscriptional level, and by structural RNAs in cell proliferation, differentiation, senescence, death, carcinogenesis, and the occurrence and development of disease [7].

The expression of lncRNAs in $\mathrm{BC}$ tissues varies considerably. Different types of lncRNAs affect BC by interfering with mRNA splicing and inducing apoptosis, $\mathrm{X}$ chromosome silencing and genomic imprinting [8]. Genomics research provides a new feasible solution for the detection, diagnosis, target selection and prognostication of BC. In this study, differentially expressed lncRNAs in $B C$ and para-carcinoma tissues were screened by gene chip technology; from these analyses, the lncRNA IGBP1-AS1 was identified, and its function was elucidated to reveal its clinical value. IGBP1-AS1 has not been studied before and therefore, its biological function as well as regulatory mechanism remained unclear. Furthermore, in this research we also confirmed the downstream targets of IGBP1-AS1. We found that miR-24-1 may be sponged by IGBP1-AS1. miR-24-1 has been reported to be involved in tumorigenesis or chemoprevention of colorectal cancer, ovarian cancer, pediatric pilocytic astrocytomas and ependymomas [9-11]. Zic family member 3 (ZIC3) is a transcription factor that has widely studied. Its function varies in several cancers [12, 13], however, in BC, it still remains unknown.

\section{Methods}

\section{Microarray profiling}

The LncRNA microarray expression profiling was carried out strictly based on the protocol provided by the manufacturer to screen the LncRNAs with varied expression levels in $\mathrm{BC}$ tissues and adjacent tissues (fold change $>1.5$ and Padj<0.05). At first the target cDNA was synthesized, labeled and purified, after which the Cyanine3-CTP labeled cRNA was hybridized with the lncRNA microarray chip. The samples were analyzed by microarray after washing. $\mathrm{R}$ project was applied for result analysis and clustering.

\section{Culture of cell lines}

The BC cell lines (HCC70 and UACC-812) and human mammary cells $(76 \mathrm{~N}-\mathrm{F} 2 \mathrm{~V})$ used in this study were gained from American Type Culture Collection (ATCC, Manassas, VA, USA). These cells were incubated in RPMI-1640 medium with 10\% fetal bovine serum (FBS) and a supplement of $100 \mu \mathrm{g} / \mathrm{L}$ streptomycin and $100 \mu \mathrm{g} / \mathrm{L}$ penicillin under the conditions of $37^{\circ} \mathrm{C}$ and $5 \% \mathrm{CO}_{2}$.

\section{Real-time quantitative polymerase chain reaction (RT-qPCR)}

The TRIzol reagent (Invitrogen, USA) was applied for total RNA extraction, and the SYBR Green Mix (Promega) was adopted for primer amplification (forward primer: AGCAGCATTTTCCTGGCTAC; reverse primer: GGTGGAGGGGGAACCCATAG). The synthesis work of the primers was provided by Zhejiang East Genecreate Biological Engineering Co., Ltd. Data analysis was conducted by using the $2^{-\Delta \Delta C t}$ method. The miR-24-1 expression was determined with the TaqMan MicroRNA Assays Kit (Applied Biosystems, USA). U6 and GAPDH were adopted as the endogenous controls for miR-24-1 and IGBP1-AS1. All the experiment procedures were conducted in triplicate.

\section{Cell transfection with lentivirus}

The pBLLV-CMV-IRES-ZsGreen IGBP1-AS1, pCMVIRES-GFP ZIC3 cDNA lentiviral plasmid for overexpression (oe) transfection and Phblv-u6-ZsGreen-Puro ZIC3 shRNA lentiviral plasmid for knock-down transfection were gained from Genelily BioTech Co., Ltd, (Shanghai, China). The transfection procedure was performed with Lipofectamine 3000 (Invitrogen). The transfected cells were cultured with puromycin $(2 \mu \mathrm{g} / \mathrm{mL})$ for 2 weeks, and the stable cells were selected. The RT-qPCR technique was adopted for transfection efficiency verification.

\section{Cell Counting Kit-8 (CCK8) assay}

After a 5-day cell incubation at $37{ }^{\circ} \mathrm{C}$ and $5 \% \mathrm{CO}_{2}$, CCK8 solution was added to each well of the 96-well plates $\left(2 \times 10^{3}\right.$ cells/well). A microplate reader was applied to detect the cell viability by measuring the absorbance value at $450 \mathrm{~nm}$. 


\section{MTT assay}

After 24, 48, 72 and 96-h cell incubation, $10 \mu \mathrm{L}$ MMT $(5 \mathrm{mg} / \mathrm{mL})$ was added to each well of the 96 -well plate $\left(1 \times 10^{4}\right.$ cells/well $)$, and the transfected BC cells were cultured for another $4 \mathrm{~h}$. After removal of the upper layer, $100 \mu \mathrm{L}$ DMSO was added. The absorbance value at $490 \mathrm{~nm}$ was detected with a microplate reader.

\section{Flow cytometry}

A previous developed protocol was applied for the flow cytometry assay [14]. Cell apoptosis was analyzed with the Annexin V-FITC early apoptosis kit, and the flow cytometer (FACScan; BD Biosciences) was adopted to analyze the IGBP1-AS1overexpressed BC cells and negative control cells, the results of which was calculated by CellQuest software (BD Biosciences).

\section{Transwell assay}

Transwell chambers $(8-\mu \mathrm{m}$ pore size; Corning Costar, USA) was used for the evaluation of cell invasion and migration. With the cells inoculated on the upper chamber, $20 \%$ serum was used as chemoattractant in the lower chamber. After 48-h incubation, methanol was used for fixation, and $0.1 \%$ crystal violet was applied for staining. Due to the slight abrasion on the upper surface of the filter, cells located on the lower surface were counted and photographed by using a microscope. All the procedures were carried out in triplicate.

\section{Wound-healing assay}

An equivalent amount of HCC70 and UACC-812 cells that was transfected with IGBP1-AS1 over-expressed plasmid and negative control (NC) plasmid were inoculated into 6-well plates. After the cells adhered to the wall and formed monolayers, a gap was drawn in the middle of the cell layer with a pipette tip. After $24 \mathrm{~h}$, a microscope was used to observe the $\mathrm{BC}$ cells that migrated into the gap area.

\section{Samples from tissue}

The $\mathrm{BC}$ tissues and adjacent normal breast tissues used in this study were gained from the surgeries carried out in the Second Affiliated Hospital of Jiaxing University from Jan 2011 to June 2017 and stored at $-80{ }^{\circ} \mathrm{C}$. Informed consents and complete clinical information was obtained from all the patients. In the end, microarray profiling was conducted with $10 \mathrm{BC}$ specimens and 6 normal breast specimens (6 paired). The survival analysis and immunohistochemical assays were conducted in the other $94 \mathrm{BC}$ specimens. The clinical procedures related to human were approved by the institutional human experiment and ethics committee of the Second Affiliated Hospital of Jiaxing University and the Affiliated Women's Hospital of Zhejiang University. All the surgical procedures were carried out strictly in line with the provisions of the Helsinki Declaration.

\section{In situ hybridization (ISH)}

The ISH assay was conducted in accordance with a previously developed method [15]. After labeling with the digoxigenin antibody (Roche, 11,093,274, 1:1000), the locked nucleic acid probe with complementary sequences of IGBP1-AS1 (custom LNA detection probe, Exiqon) was synthesized. The staining intensity was reviewed by 2 independent pathologists who were blinded to the study design.

\section{Luciferase reporter assays}

The pmirGLO-IGBP1-AS1-wt reporter vector was prepared by cloning the IGBP1-AS1 cDNA with miR-24-1 predictive binding site into the pmirGLO Dual-Luciferase miRNA Target Expression Vector (Promega). A similar procedure was carried out with the IGBP1-AS1 cDNA with point mutations of the miR-24-1 seed region binding site to form the pmirGLO-IGBP1-AS1-Mut reporter vector. Then the miR-24-1 and miR-NC were transfected with the vectors into the HEK-293FT cells by using Lipofectamine 3000 (Invitrogen).

The analyses on the websites UCSC (http://genom e.ucsc.edu/) and JASPAR (http://jaspar.genereg.net/) indicated that the ZIC3 protein might bind to the DNA sites of IGBP1-AS1. The IGBP1-AS1 recombinant luciferase reporter vector with truncated or mutated binding sites was constructed and co-transfected into the HCC70 cells with the ZIC3 expression vector to verify the specific sites of ZIC3 protein binding to IGBP1-AS1 DNA. After $48 \mathrm{~h}$ of transfection, the luciferase reporter assay was then carried out with a luciferase assay kit (K801-200, Biovision, USA) following the manufacturer's instruction. With renilla luciferase as an internal reference gene, the activation degree of the target reporter gene was compared based on the ratio of the relative luciferase unit (RLU) of firefly luciferase assay divided by the RLU of Renilla luciferase assay.

\section{RNA immunoprecipitation (RIP)}

The trial was carried out strictly based on the protocol of EZMagna RIP Kit (Millipore) provided by the manufacturer. In brief, after cell lysis with the complete RNA immunoprecipitation (RIP) lysis buffer, extract of the $\mathrm{BC}$ cells was incubated together with anti-argonaute 2 (AGO2) or control anti-IgG antibody conjugated magnetic beads at $4{ }^{\circ} \mathrm{C}$ for $6 \mathrm{~h}$. The purified RNA was 
evaluated with the RT-qPCR after removing the proteins of the beads.

\section{RNA pull-down assay}

The 3 'end biotinylated miR-24-1 (TGCCTACTGAGC TGATATCAGT) or miR-24-1-mut (TGCCTACTCAGC TGATATCAGT) $(20 \mathrm{nmol} / \mathrm{L})$ were transfected into the HCC70 cells. After $24 \mathrm{~h}$ of incubation with streptavidincoated magnetic beads (Life Technologies), pull down assay was conducted in a biotin-coupled RNA complex. Finally, the IGBP1-AS1 abundance was calculated based on the RT-qPCR results.

\section{Western blot assay}

The RIPA lysis buffer with PMSF was applied for the extraction of the total protein in tissues and cells, which were incubated in an ice bath for $30 \mathrm{~min}$. The lysate was centrifuged for 10 min under the condition of $4{ }^{\circ} \mathrm{C}$ and $8000 \mathrm{~g}$ to collect the supernatant. The SDS-PAGE gel electrophoresis was performed with all the samples, and the separated protein was transferred to PVDF membrane. $5 \%$ skimmed milk was used for membrane blocking. After $1 \mathrm{~h}$ room-temperature blocking, the membrane was incubated together with primary sheep anti-ZIC3 (1:1000, ab215063, Abcam, UK) and rabbit anti-GAPDH (1:2500, ab9485, Abcam, UK) at $4{ }^{\circ} \mathrm{C}$ overnight, followed by 1-h incubation with HRP-labeled secondary antibody. Afterwards, the membrane was rinsed with TBST, and the proteins were visualized by using the ECL Fluorescence Detection Kit (Cat. No. BB-3501, UK) and photographed by Bio-Rad Image Analysis System (BIO-RAD, USA). The software of Quantity One v4.6.2 was adopted for quantification. The protein expression levels were presented as the relative gray values of the interested protein band and the GAPDH protein band. All the procedures were performed in triplicate and the final results were presented as mean values.

\section{Chromatin immunoprecipitation (ChIP)}

Formaldehyde was used for cell fixation for $10 \mathrm{~min}$. The chromatin fragments were obtained by cell sonication, and the supernatant was collected after centrifugation. The negative control rabbit IgG (ab109489, 1:300, Abcam, Shanghai, China) and the target proteinspecific antibody ZIC3 (sc-101201, 1:1000, Santa Cruz Biotechnology, Shanghai, China) were added to fully incubate supernatant at $4{ }^{\circ} \mathrm{C}$ overnight. The protein agarose/Sepharose were used for DNA-protein complex precipitation, which was centrifugated at $12,000 \mathrm{~g}$ for $5 \mathrm{~min}$, followed by discard of the supernatant. After washing off the non-specific complex, cross-linking was conducted at $65{ }^{\circ} \mathrm{C}$ overnight. The DNA fragment was extracted, purified and recovered by phenol/chloroform.
The primer was designed to amplify which contains the site of ZIC3 binding to the IGBP1-AS1 DNA promoter (F: 5'-CTTCATGGTGCAGGGTGCTA-3', R: 5'-TGC ATGTGGTTGTGCTCAGA- $3^{\prime}$ ). The amplified product was 775 bp long. A Distal primer (a primer that amplifies the sequence away from the IGBP1-AS1 DNA promoter region) was designed as a negative control for the site primer (F: 5'-AGCTCATTTCTCCCCTTGCC-3', R: $5^{\prime}$-TCTCTACTCCCACCAGAGGC- $3^{\prime}$ ) and the amplified product was $384 \mathrm{bp}$ long. Using the recovered DNA fragment as an amplification template, site primers and Distal primers were added respectively to perform RTqPCR to verify whether the site of IGBP1-AS1DNA was the binding site of transcription factor ZIC3.

\section{Animal trials}

The BALB/c-nude mice aged 4 to 5 weeks were obtained from Shanghai SLAC Laboratory Animal Co., Ltd. All the laboratory procedures related to animals were approved by the institutional animal care and use committees of both institutions. The concentration of the stably infected cells was adjusted to $5 \times 10^{6}$ cells $/ \mathrm{mL}$ after being suspended in 50\% Matrigel (BD Biosciences, Bedford, MA). $0.4 \mathrm{~mL}$ cell suspension $\left(2 \times 10^{6}\right.$ cells $)$ was administrated to the nude mice by means of subcutaneous injection at the left axilla area. The tumor size was examined every 5 days, and volume of the tumor was calculated with the formula of length $\times$ width $^{2} \times 0.5$. All nude mice were euthanized, and the tumors were weighed 30 days later.

The mice were submitted toa right lateral flank incision after anesthesia to construct the abdominal metastasis model. $100 \mu \mathrm{L}$ Hank's balanced salt solution containing HCC70-Luc-vector and HCC70-Luc cells $\left(5 \times 10^{6}\right.$, transfected with Lv-IGBP1-AS1, miR-24-1 mimic, Lv-IGBP1$\mathrm{AS} 1+\mathrm{Si}-\mathrm{ZIC} 3$ or $\mathrm{Lv}-\mathrm{ZIC} 3+\mathrm{miR}-24-1$ mimic) was administrated into the right abdominal cavity by injection. 4 weeks after the operation, the bioluminescence images were collected by the Interactive Video Information System (IVIS).

\section{Statistical methods}

All the numerical measurements were presented as mean \pm SD. The student's $t$ test and one-way ANOVA test were applied for the evaluation of group differences according to the data nature. The overall survival time was defined as the period between the diagnosis and death of any cause, and the progression-free time duration started from the beginning of treatment and ended when disease progression occurred. Log-rank test and Cox's regression analysis were applied for univariate and multivariate survival analyses. $P<0.05$ was considered as the standard for statistical significance. All the analyses 
row $\min$

a ${ }^{\mathrm{A}}$

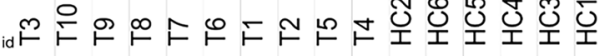

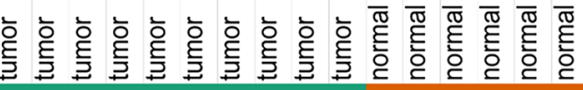

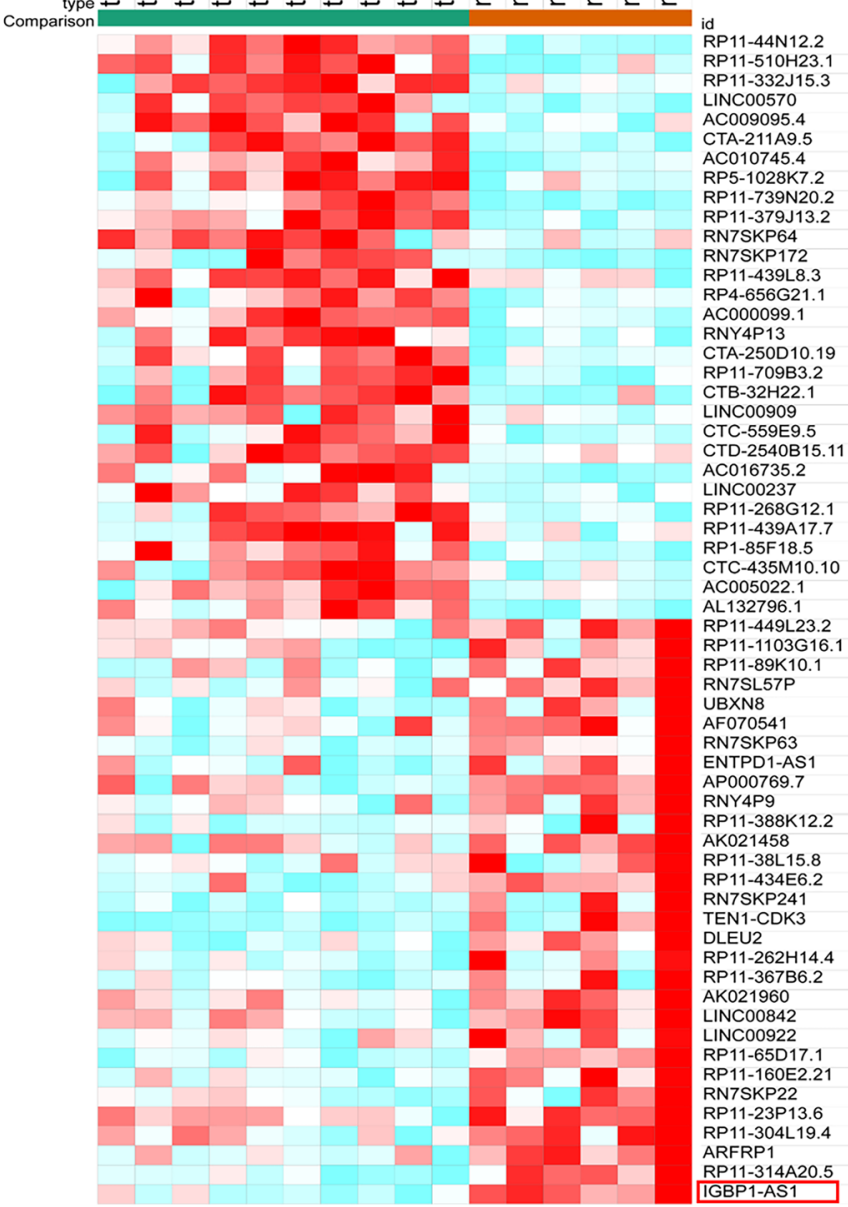

b

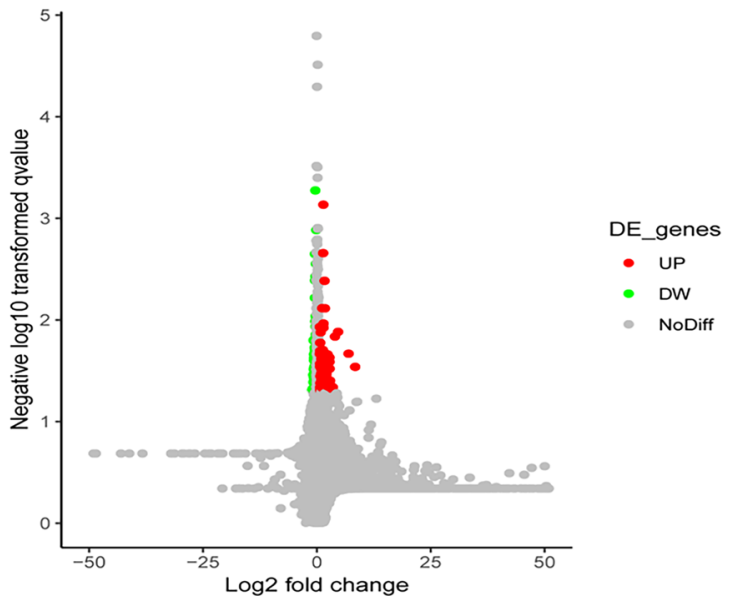

Fig. 1 Down-regulation of the LnCRNA IGBP1-AS1 in BC tissues was observed. a The heat map presented the 60 IncRNAs with differed expression levels in BC specimens and normal adjacent specimens (fold change $>1.5 ; P<0.05$ ). $\mathbf{b}$ The volcano plot of the LncRNAs with the colored dots representing the $\operatorname{LnCRNAs}$ with $\log _{2}$ (fold change) $>0.66$ and $P<0.05$ 
were conducted with SPSS 22.0 and the imagines were drawn with GraphPad Prism 7.0.

\section{Results \\ Significant down-regulation of the LncRNA IGBP1-AS1 in $\mathrm{BC}$ tissues}

In total 60 lncRNAs with differed expression levels were identified (Fig. 1a, b). IGBP1-AS1 localized on chromosome Xq13.1 was significantly decreased in BC tissues (Fig. 1a)

\section{Inhibitory effect on viability and invasion of $B C$ cells induced by IGBP1-AS1}

The RT-qPCR demonstrated that the expression level of IGBP1-AS1 in BC cell lines was significantly down-regulated compared to the normal human mammary cells $(76 \mathrm{~N}-\mathrm{F} 2 \mathrm{~V})(P<0.05)($ Fig. $2 \mathrm{a})$. The cell proliferation and viability (obtained through CCK8 and MTT assays) in the IGBP1-AS1 overexpressed group were reduced compared with the negative control group (Fig. 2c, d). Furthermore, the cell cycle was inhibited (Fig. 2e) and cell apoptosis was enhanced in the IGBP1-AS1 overexpressed group (Fig. 2f). The transwell assay and wound-healing assay indicated that the increased expression of IGBP1AS1 could have significant inhibitory impact on the cell's invasive capacity $(P<0.05)$ (Fig. $2 \mathrm{~g}, \mathrm{~h})$.

\section{Positive impact of IGBP1-AS1 on the survival outcomes of $\mathrm{BC}$ patients}

There were $94 \mathrm{BC}$ tissues with complete clinical information included in survival analysis. According to the ISH assay, the expression level of IGBP1-AS1 in BC tissues was lower compared to the adjacent normal tissues (Fig. 2i). The expression level of IGBP1-AS1 was classified based on the staining intensity score in ISH (score 0-2: low expression; score 3-4: high expression). Table 1 contains the patient characteristics at baseline. The median overall survival (OS) was 40 months in the high expression of IGBP1-AS1 group and 14 months in the low expression of IGBP1-AS1 group (Fig. 2j). The median progression-free survival (PFS) of the low expression group was 10 months, which was significantly longer than that of the high expression group $(P$ value $<0.001)$
(Fig. 2k). The multivariate analyses verified that IGBP1AS1 expression in BC could be an independent risk factor for the OS (HR: 3.102, 1.365 to 6.526, $P=0.005$ ) (Table 2). Another independent factor is disease staged at II, III or IV. With respect to the PFS, lower IGBP1-AS1 expression level could also lead to worse survival outcome regarding disease progression (HR: 4.472, 1.565 to 13.926, $P=0.009$ ) (Table 3). These results indicate that IGBP1-AS1 could have a positive effect on the survival outcomes in $\mathrm{BC}$ patients.

\section{Verification of impact of IGBP1-AS1 in vivo}

In our study, we found that tumor tissues with highly expressed IGBP1-AS1tended to be smaller in volume and lower in weight (Fig. 3a). To validate the impact of IGBP1-AS1 on metastases, we performed an abdominal metastasis model. According to the bioluminescence imaging, overexpressed IGBP1-AS1 could significantly inhibit cell invasion in vivo $(P<0.05)($ Fig. $3 \mathrm{~b})$.

\section{IGBP1-AS1 targeted and regulated miR-24-1}

The target miRNA with reversed complementary sequence of IGBP1-AS1 was predicted by bioinformatics (miRcode http://www.mircode.org/), and miR-24-1 was selected for further research. Based on the results of the luciferase reporter assay of pmirGLO-IGBP1-AS1wt reporter vector, the luciferase activity in the HCC70 cells transfected with miR-24-1 was significantly reduced compared to the cells transfected with miR-NC. With respect to the pmirGLO-IGBP1-AS1-mut reporter vector, the luciferase activity of the two groups did not differ from each other (Fig. 4a). The results of the RIP assay indicated that IGBP1-AS1 and miR-24-1 were particularly enriched in AGO2 compared to anti-IgG immunoprecipitates (Fig. 4b). In addition, the RNA pull-down assay suggested that the level of IGBP1-AS1 was higher in the miR-24-1-wt than in the miR-24-1-mut (Fig. 4c). Furthermore, the RT-qPCR demonstrated a decreased expression of miR-24-1 in BC cells with overexpressed IGBP1-AS1 (Fig. 4d). The weakened expression of IGBP1 (Fig. 4e) and the enhancement of cell proliferation (Fig. 4f) and invasion (Fig. 4g) caused by the miR-24-1

\footnotetext{
(See figure on next page.)

Fig. 2 In-vitro inhibitory effect of the IGBP1-AS1 on BC cell proliferation. a The RT-qPCR indicated the expression of IGBP1-AS1 in BC cell lines (HCC70 and UACC-812) relative to human mammary cells (76N-F2V). b The RT-qPCR demonstrated up-regulated efficiency of IGBP1-AS1 in BC cells. ${ }^{*} P$ value $<0.01$. c, $\mathbf{d}$ Assessment of cell proliferation and viability by CCK8 and MTT assays. ${ }^{*} P$ value $<0.01$. e The FACS analysis indicated overexpression of IGBP1-AS1 may reduce the number of cells in the G2/M phase. ${ }^{*} P$ value $<0.01$. $\mathbf{f}$ Increased expression level of IGBP1-AS1 could promote the apoptosis of $B C$ cells according to the Annexin $V$ assay. ${ }^{*} P$ value $<0.01 . \mathbf{g}, \mathbf{h}$ The results of the transwell assay and wound-healing assay performed with HCC70 and UACC-812 cells transfected with LV-IGBP1-AS1 or LV-NC. ${ }^{* *} P$ value $<0.01$ vs. control. i The expression level of IGBP1-AS1 in BC tissues and adjacent normal tissues according to the ISH assay. $\mathbf{j}, \mathbf{k}$ Comparison of the Kaplan-Meier curves of the BC patients with respect to OS rate and PFS rate respectively
} 


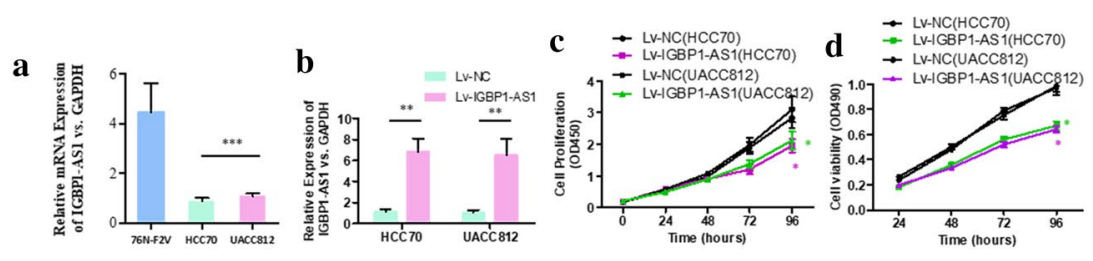

e
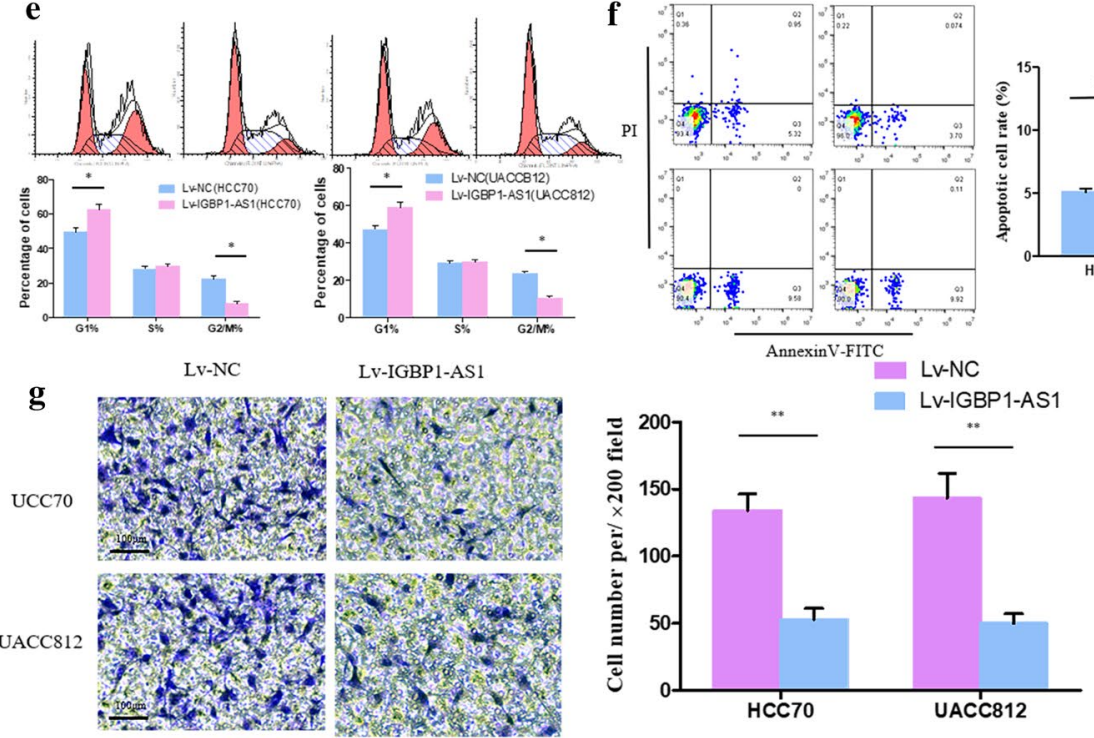

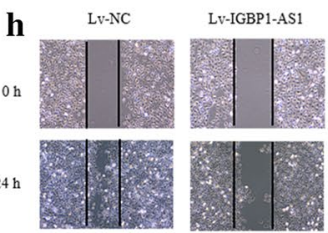

UCC70

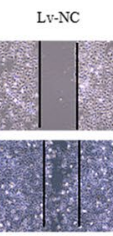

UACC812

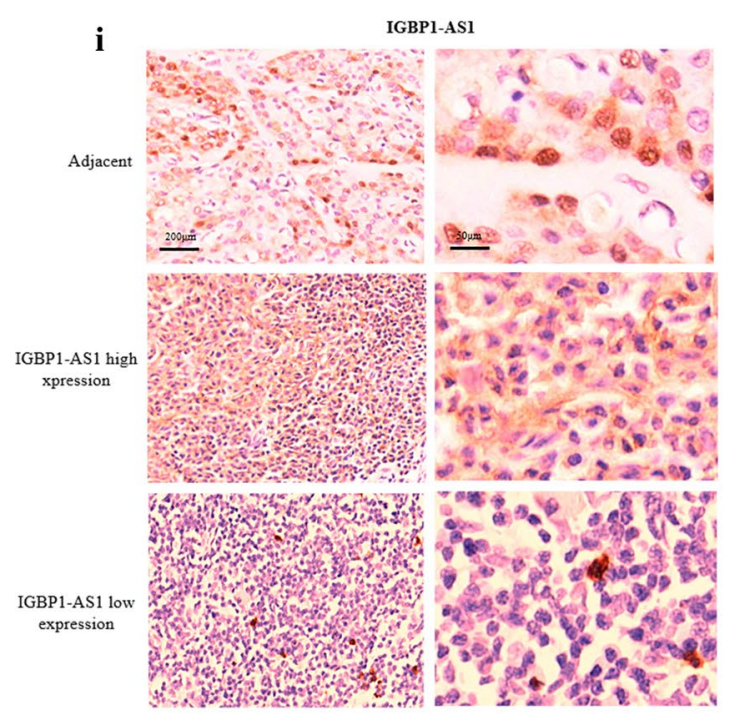

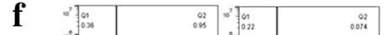
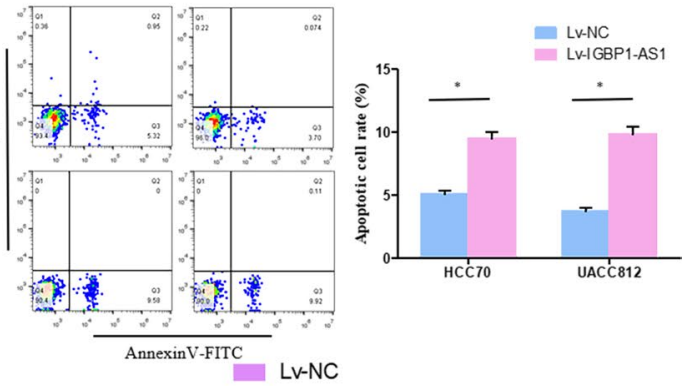
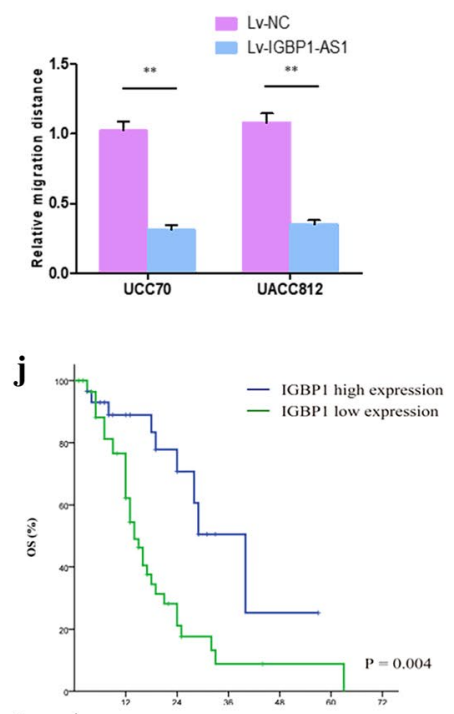

k

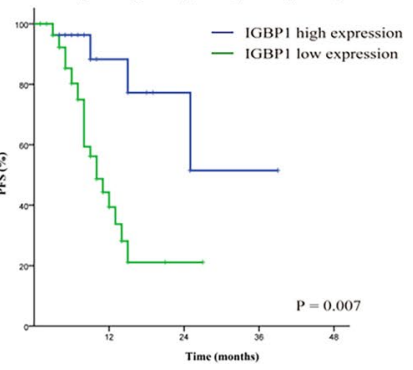


Table 1 Clinical characteristics of patients with breast cancer

\begin{tabular}{|c|c|c|c|c|}
\hline Characteristic & All patients & $\begin{array}{l}\text { IGBP1-AS1 } \\
\text { High expression }\end{array}$ & $\begin{array}{l}\text { IGBP1-AS1 } \\
\text { Low expression }\end{array}$ & $P$-value \\
\hline Total & 94 & 30 & 64 & \\
\hline Age (years) & & & & 0.382 \\
\hline$<60$ & 74 & 22 & 52 & \\
\hline$\geq 60$ & 20 & 8 & 12 & \\
\hline \multicolumn{5}{|l|}{ Menopause } \\
\hline No & 69 & 23 & 46 & 0.624 \\
\hline Yes & 25 & 7 & 18 & \\
\hline \multicolumn{5}{|l|}{ Invasion depth } \\
\hline $\mathrm{T} 1$ & 13 & 3 & 10 & 0.461 \\
\hline $\mathrm{T} 2-4$ & 81 & 27 & 54 & \\
\hline \multicolumn{5}{|c|}{ Lymph node metastasis } \\
\hline No & 38 & 9 & 29 & 0.158 \\
\hline $\mathrm{N} 1-3$ & 56 & 21 & 35 & \\
\hline \multicolumn{5}{|l|}{ Stage } \\
\hline I & 30 & 7 & 23 & 0.222 \\
\hline$\|/\| I / I V$ & 64 & 23 & 41 & \\
\hline \multicolumn{5}{|l|}{ Histologic grade } \\
\hline$|/| \mid$ & 58 & 20 & 38 & 0.498 \\
\hline III & 36 & 10 & 26 & \\
\hline \multicolumn{5}{|l|}{ Histologic type } \\
\hline Ductal & 81 & 27 & 54 & 0.461 \\
\hline Lobular & 13 & 3 & 10 & \\
\hline \multicolumn{5}{|l|}{ ER } \\
\hline Negative & 47 & 13 & 34 & 0.376 \\
\hline Positive & 47 & 17 & 30 & \\
\hline \multicolumn{5}{|l|}{ PR } \\
\hline Negative & 63 & 21 & 42 & 0.674 \\
\hline Positive & 31 & 9 & 22 & \\
\hline \multicolumn{5}{|l|}{ HER2 } \\
\hline Negative & 46 & 12 & 34 & 0.235 \\
\hline Positive & 48 & 18 & 30 & \\
\hline
\end{tabular}

ER estrogen receptor, $P R$ progesterone receptor, HER2 human epidermal growth factor receptor 2

mimic could be largely reversed by overexpressed IGBP1AS1 in the rescue experiments.

\section{In-vivo/in vitro modulation effect of the Lnc IGBP1-AS1/} miR-24-1/ZIC3 axis on the invasion and proliferation of $B C$ cells

The binding sites in several genes' $3^{\prime}$-UTR with miR24-1 were predicted by bioinformatics (Targetscan 7.2; miRDB; miRTarBase). Zic Family Member 3 (ZIC3) was considered as the target gene based on the screening of RT-qPCR (Fig. 5a). The luciferase assay showed that the 3'-UTR of wild-type ZIC3 had the ability to significantly inhibit the luciferase activity of the cells transfected with miR-24-1, though it had no impact on the cells transfected with miR-NC. In addition, 3'-UTR of mutant-type
ZIC3 did not significantly influence the luciferase activity of the cells transfected with miR-24-1 (Fig. 5b). It was confirmed in the RNA pull-down assay that the content of ZIC3 3'-UTR was higher in the wild-type miR-24-1 compared to the mutant-type miR-24-1 with mutant ZIC3 3'-UTR binding site (Fig. 5c). The western blot assay and RT-qPCR suggested that the level of miR-24-1 could have an impact on the mRNA and protein expression in ZIC3 (Fig. 5d, e). Moreover, the up-regulation of miR-24-1 in BC cells had reversal effect on the inhibition of cell viability (Fig. 5f), invasion and migration (Fig. 5g) induced by ZIC3 overexpression. The HCC70 cells that transfected with NC, Lv-IGBP1-AS1, miR-24-1 mimic, Lv-IGBP1-AS1 + Si-ZIC3 and miR-24-1 mimic + LvZIC3 were administrated into the abdominal cavity of 
Table 2 Univariate and multivariate analysis of breast cancer patients on overall survival

\begin{tabular}{|c|c|c|c|c|}
\hline \multirow[t]{2}{*}{ Variable } & \multicolumn{2}{|l|}{ Univariate analysis } & \multicolumn{2}{|c|}{ Multivariate analysis } \\
\hline & $\mathrm{HR}(95 \% \mathrm{Cl})$ & $P$ & $\mathrm{HR}(95 \% \mathrm{Cl})$ & $P$ \\
\hline Age ( $\geq 60$ vs. $<60$ years) & $1.033(0.997-1.062)$ & 0.057 & & \\
\hline Menopause (yes vs. no) & $1.107(0.620-1.875)$ & 0.134 & & \\
\hline Stage (II/III/IV vs. I) & $2.726(1.256-4.789)$ & 0.021 & $2.035(1.477-3.329)$ & 0.011 \\
\hline IGBP1-AS1 expression (low vs. high) & $3.144(1.494-6.616)$ & 0.003 & $3.102(1.365-6.526)$ & 0.005 \\
\hline Histologic grade (III vs. I/II) & $1.373(0.384-2.513)$ & 0.463 & & \\
\hline Histologic type (lobular vs. ductal) & $1.208(0.507-3.391)$ & 0.601 & & \\
\hline ER (yes vs. no) & $0.827(0.384-2.731)$ & 0.379 & & \\
\hline PR (yes vs. no) & $0.595(0.277-1.982)$ & 0.301 & & \\
\hline HER2 (yes vs. no) & $1.282(0.893-2.191)$ & 0.115 & & \\
\hline
\end{tabular}

Italic values indicate significance of $P$ value $(P<0.05)$

$E R$ estrogen receptor, $P R$ progesterone receptor, HER2 human epidermal growth factor receptor 2

Table 3 Univariate and multivariate analysis of breast cancer patients on progress free survival

\begin{tabular}{|c|c|c|c|c|}
\hline \multirow[t]{2}{*}{ Variable } & \multicolumn{2}{|l|}{ Univariate analysis } & \multicolumn{2}{|l|}{ Multivariate analysis } \\
\hline & $\mathrm{HR}(95 \% \mathrm{Cl})$ & $P$ & $\mathrm{HR}(95 \% \mathrm{Cl})$ & $P$ \\
\hline Age ( $\geq 60$ vs. $<60$ years) & $1.103(0.908-1.462)$ & 0.094 & & \\
\hline Menopause (yes vs. no) & $1.002(0.521-1.745)$ & 0.173 & & \\
\hline Stage (II/III/IV vs. I) & $1.957(1.052-2.789)$ & 0.032 & $1.713(0.9524-2.329)$ & 0.067 \\
\hline IGBP1-AS1 expression (low vs. high) & $4.602(1.559-13.588)$ & 0.006 & $4.472(1.565-13.926)$ & 0.009 \\
\hline Histologic grade (III vs. I/II) & $1.076(0.448-2.273)$ & 0.416 & & \\
\hline Histologic type (lobular vs. ductal) & $1.198(0.407-3.131)$ & 0.592 & & \\
\hline ER (yes vs. no) & $1.072(0.568-1.591)$ & 0.214 & & \\
\hline PR (yes vs. no) & $1.315(0.650-2.081)$ & 0.324 & & \\
\hline HER2 (yes vs. no) & $1.482(0.749-2.438)$ & 0.195 & & \\
\hline
\end{tabular}

Italic values indicate significance of $P$ value $(P<0.05)$

$E R$ estrogen receptor, $P R$ progesterone receptor, HER2 human epidermal growth factor receptor

the $\mathrm{BALB} / \mathrm{c}$-nude mice by injection. The results observed in the abdominal metastasis model were similar to those in vitro (Fig. 5h).

\section{IGBP1-AS1 might be further up-regulated by recruiting its downstream target: transcription factor ZIC3}

The target gene ZIC3 was well-known as a transcription factor. To determine whether it could bind to IGBP1AS1 at the promoter region, we searched and predicted the most possible site of ZIC3 protein binding to IGBP1AS1 DNA by UCSC (http://genome.ucsc.edu/) and JASPAR (http://jaspar.genereg.net/) (Fig. 6a). The RIP assay verified the binding of IGBP1-AS1 to ZIC3 (Fig. 6b). Compared with the IgG, the binding of ZIC3 to IGBP1AS1 was notably increased $(P<0.05)$, and the anti-ZIC3 antibody could precipitate IGBP1-AS1, indicating that
IGBP1-AS1 can form a complex with ZIC3. Furthermore, the luciferase reporter assay (Fig. 6c) demonstrated a significant up-regulation in the ability of oe-ZIC3 to activate IGBP1-AS1 $(\mathrm{P}<0.05)$. When the site was truncated or mutated, luciferase activity was the same compared to the cells transfected with oe-NC. These results confirmed that the predicted site was indeed the ZIC3 protein binding to IGBP1-AS1 DNA site. Next, the binding ability of ZIC3 to the IGBP1-AS1 DNA binding site was validated by ChIP assay in HCC70 cells (Fig. 6d). The amount of amplification products obtained from site primers in the ZIC3 group was larger than from the Distal primers in IgG group, while there was no significant difference in the amount of amplification products between the two pairs of primers in the IgG group. The results indicated that the predicted site of IGBP1-AS1 DNA was indeed 

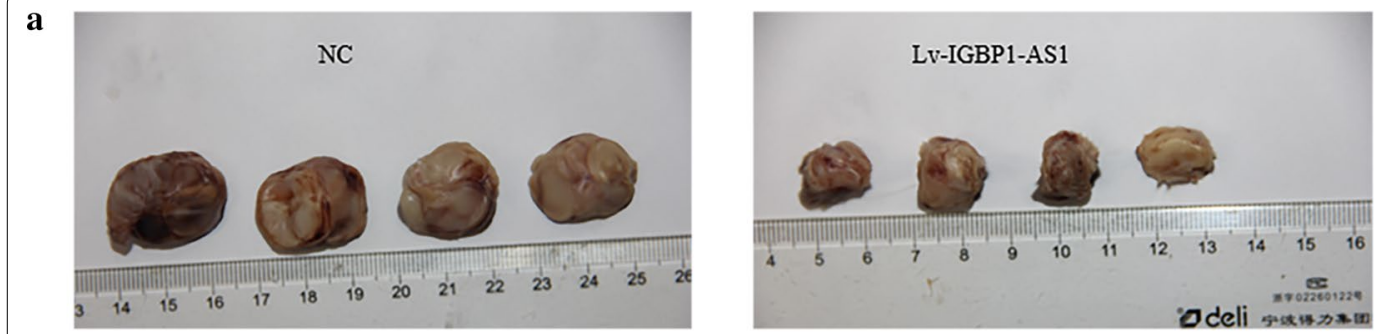

$\because \mathrm{NC}$

$\rightarrow$ LV-IGBP1-AS1
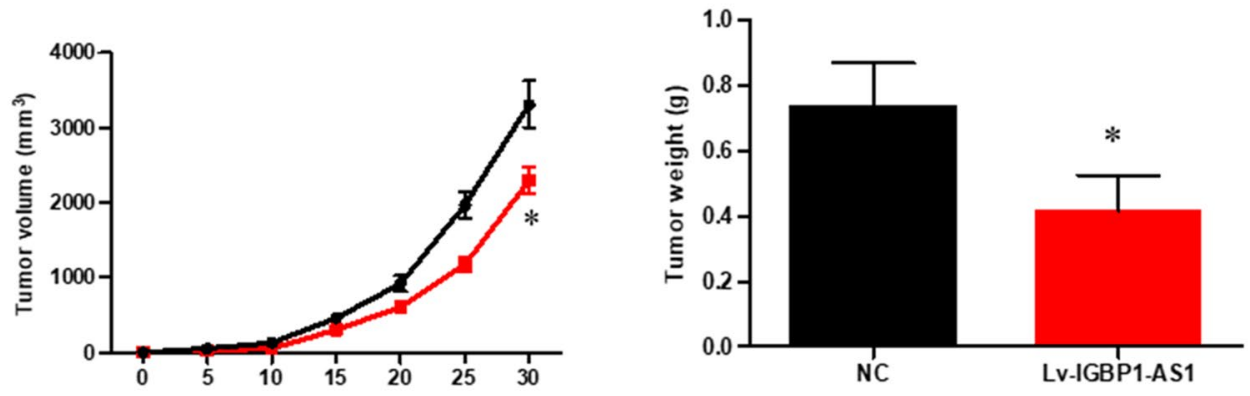

b
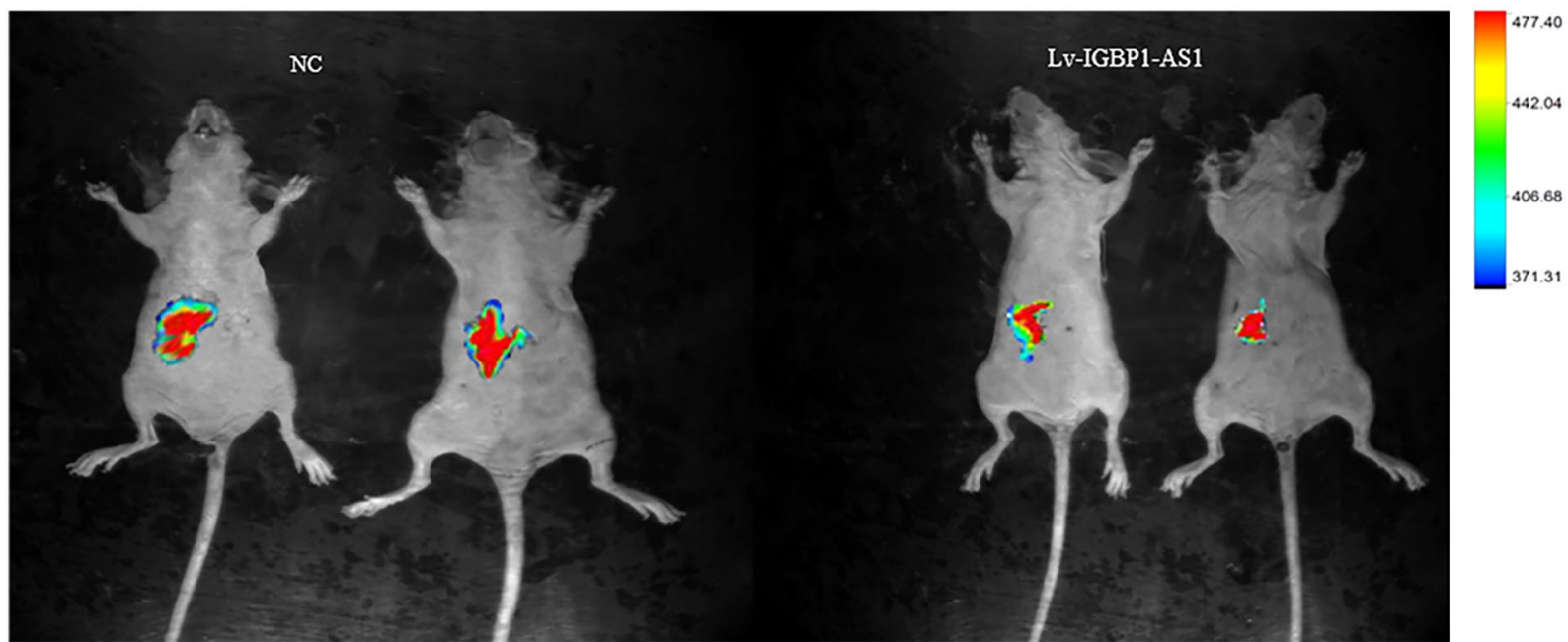

Fig. 3 In-vivo effect of the IGBP1-AS1 on metastasis and proliferation. a Animal trial performed by means of a subcutaneous injection of the HCC70 cells. b The luciferase activity of the HCC70 cells and control cells collected by bioluminescent imaging. ${ }^{* *} P$ value $<0.01$

the binding site to transcription factor ZIC3. It could be deduced that when IGBP1-AS1 up-regulates the level of ZIC3 via suppressing miR-24-1, the elevated ZIC3 could synchronously promote the expression of IGBP1-AS1. Synergistic action might be observed.

\section{Discussion}

Previously, studies have identified some BC-related lncRNAs. HOTAIR is a kind of lncRNA that is found in human fibroblasts and is transcribed in antisense, and its expression is abnormally elevated in breast cancer and its metastases. The expression of HOTAIR results in 


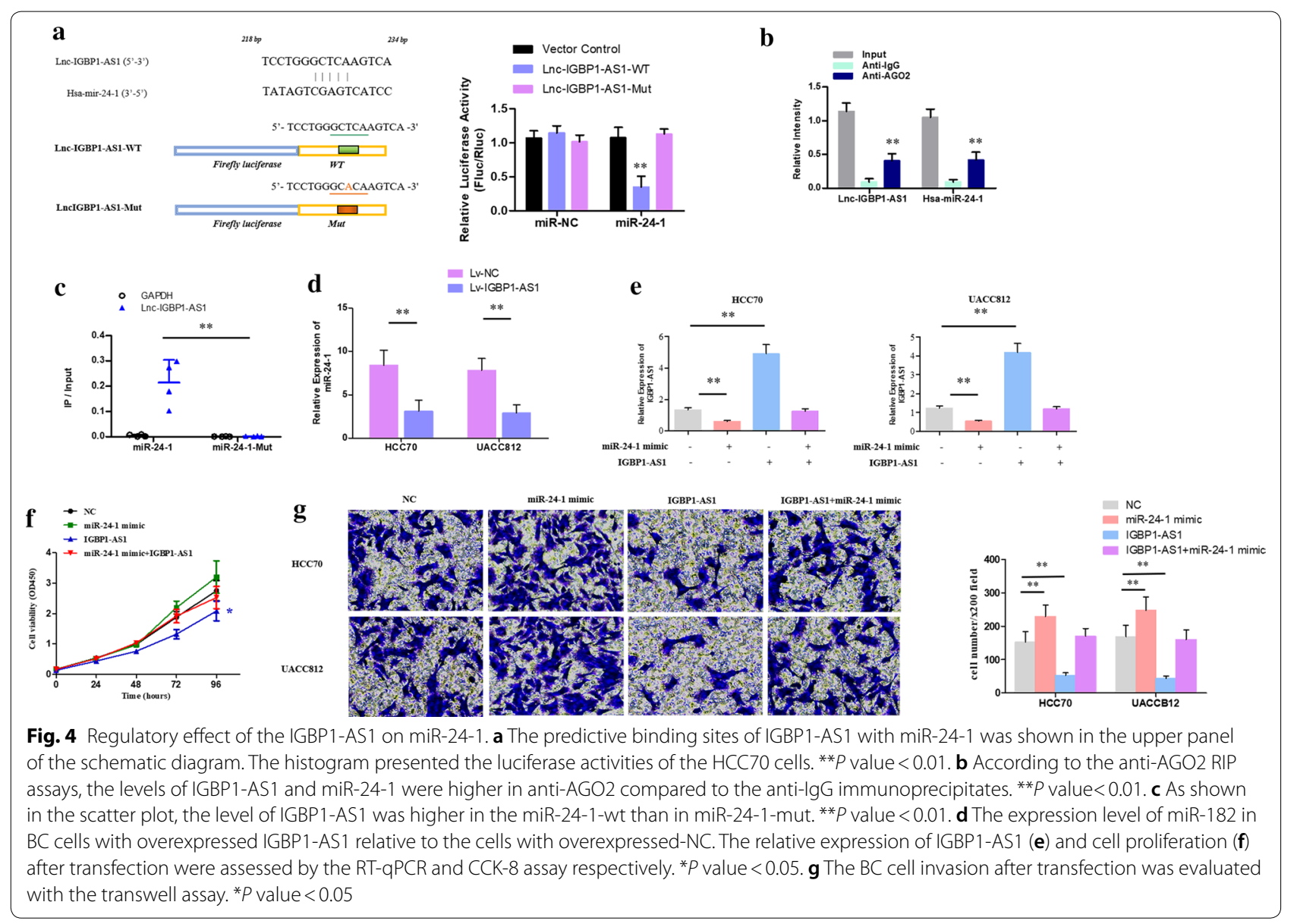

increased invasion of breast cancer cells, which is closely related to the progression of breast cancer [16], thus leading to decreased survival and poor prognosis. HOTAIR is also a prognostic indicator for breast cancer. GokmenPolar et al. [17] believed that its use for prognostication is only suitable for breast cancer patients with ER-negative disease and lymph node metastases. Therefore, the significance of HOTAIR as a prognostic indicator for breast cancer is relatively limited. Of course, there are different opinions. Milevskiy et al. [18] found that HOTAIR was coexpressed with FOXA1 and FOXM1 in HER2 receptorrich tumors when analyzing breast cancer-related gene expression data. Li et al. [19] screened a group of lncRNA microarrays involving trastuzumab-resistant SKBR-3/Tr cells and found that the expression of GAS5 is decreased in breast cancer patients and SKBR-3/Tr cells after trastuzumab treatment, demonstrating that trastuzumab can reduce the expression of GAS5. It was found that lapatinib upregulated the expression of GAS5 by inhibiting the PI3K/Akt/mTOR signaling pathway. GAS5 acts as a competitive endogenous RNA (ceRNA) of miR-21, competitively binding to miR-21 to increase PTEN and promote proliferation and metastasis of trastuzumabresistant breast cancer cells. This process improves the therapeutic resistance of breast cancer to trastuzumab. Finally, it has been shown that GAS5 can improve the therapeutic resistance of HER2-positive breast cancer to trastuzumab, suggesting that GAS5 can be used as a novel prognostic indicator and candidate drug target for HER2positive breast cancer and can improve the effect of treatment on trastuzumab-resistant patients.

Our findings suggest that expression of the lncRNA IGBP1-AS1 was remarkably lower in BC tissues and cell lines than in adjacent normal tissues. The in vivo imaging system showed that BC cells overexpressing IGBP1-AS1 had less invasive ability in the peritoneal cavity of mice than the control cells, which was also confirmed in vitro. At the same time, a retrospective analysis was performed on tumor tissues from 94 patients with BC. Multivariate analysis showed that IGBP1-AS1 expression was an independent risk factor affecting both OS and PFS and was not affected by disease stage. The median OS and PFS 


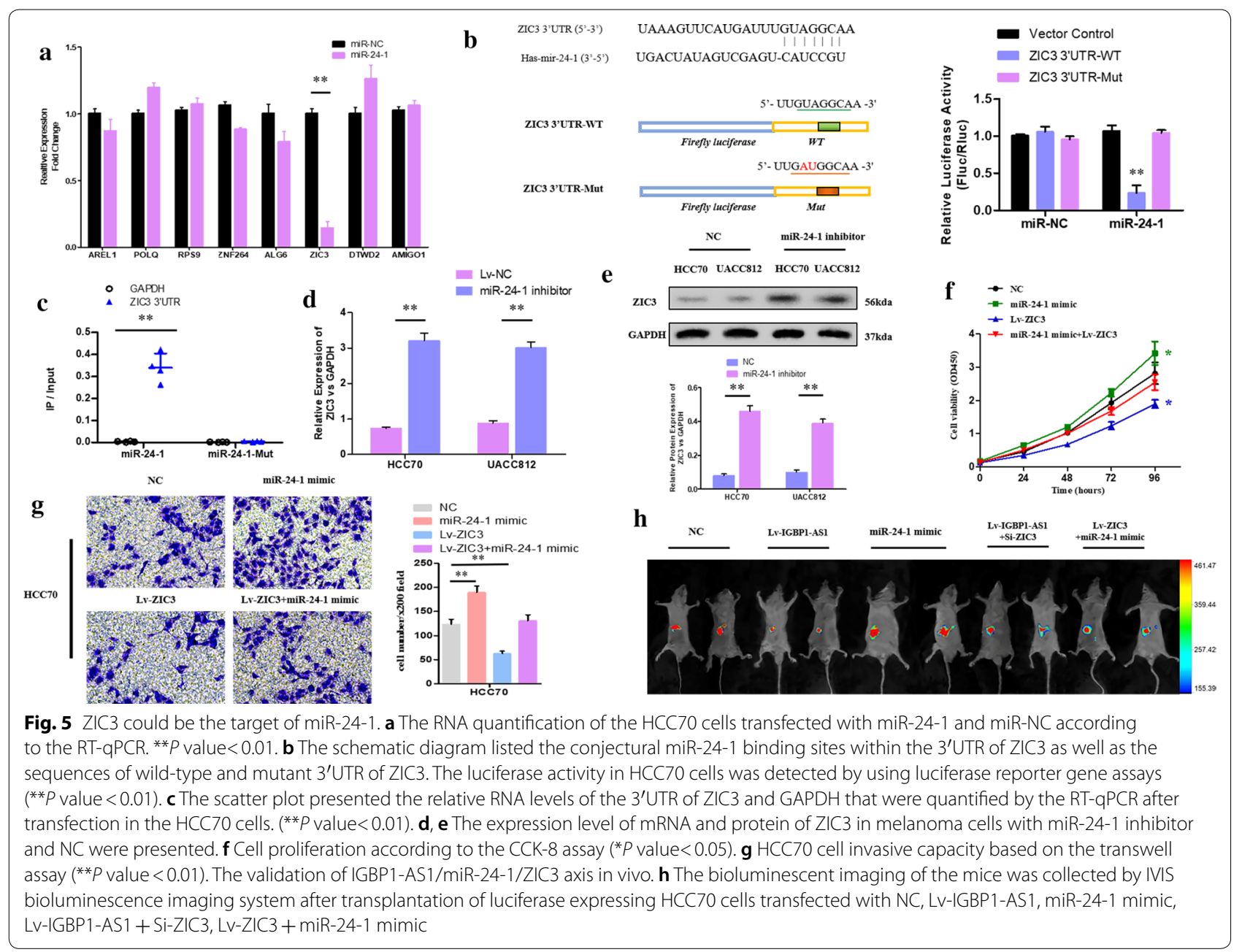

in the IGBP1-AS1high expression group were remarkably higher than those in the IGBP1-AS1low expression group, indicating that IGBP1-AS1 has clinical value in the prognosis of $\mathrm{BC}$. Loss-of-function assays were not performed in this study as the level of IGBP1-AS1 in BC were relatively low. Remarkable changes in phenotypes may not be observed when knock-down was conducted. The rescue experiments have proved that to some extent. Further mechanism research has identified that LncRNA IGBP1-AS1/miR-24-1/ZIC3 axis has significant impact on proliferation and invasion ability of $\mathrm{BC}$ cells in vitro and in vivo. miR-24-1 had never been reported in BC before. According to our results, it may act as an oncogene in $\mathrm{BC}$, which was in accord with studies in colorectal cancer and ovarian cancer $[9,10]$. It was not the first time that miR-24-1 was involved in loop-feedback regulation [20]. Some members of the ZIC family has been reported as tumor suppressor in gastric cancer including ZIC3 [12]. Interestingly, we found that ZIC3 also binds to IGBP1-AS1 at the promoter region making the axis a ring loop which is rare. It is a favorable feedback regulation in $\mathrm{BC}$ which may present another new therapeutic strategy. Although this regulation might be weakened or compensated in the human body. There were also some limitations. Sample size for survival analysis needs to be enlarged greatly to make bigger clinical impact as well as performing a prospective study. The signaling pathways that were affected by the LncRNA IGBP1-AS1/miR-24-1/ ZIC3 loop need to be further explored.

\section{Conclusion}

Expression of the LncRNA IGBP1-AS1 is decreased in $\mathrm{BC}$, and in vitro and in vivo experiments confirm that its biological function in $\mathrm{BC}$ serves to resist tumor proliferation and invasion. The LncRNA IGBP1-AS1/miR24-1/ZIC3 loop may be regarded as new therapeutic targets as it regulates proliferation and invasion of $\mathrm{BC}$. However, the lncRNA IGBP1-AS1 is still rarely studied. An in-depth study of its biological functions and 

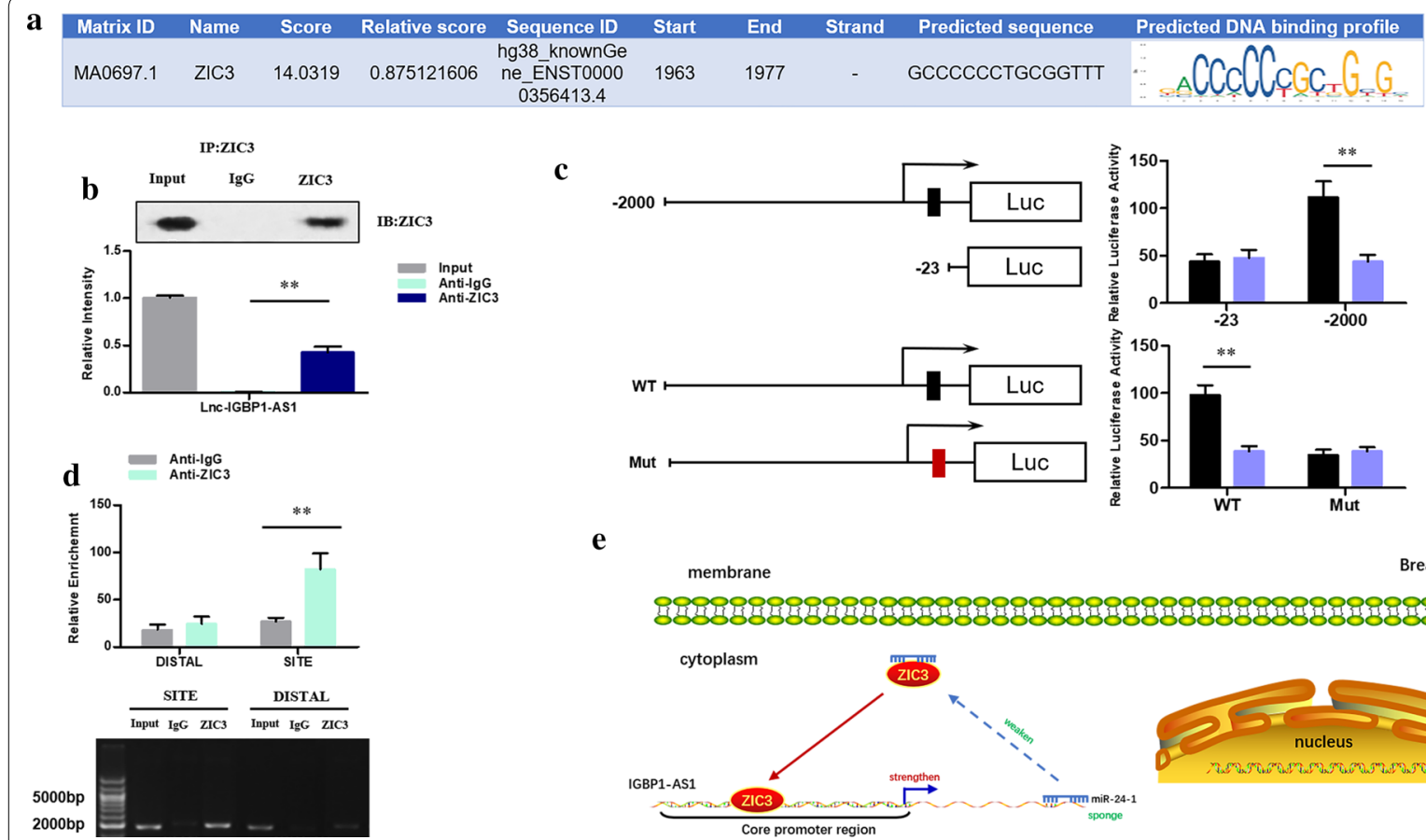

Fig. 6 a Predicted site where ZIC3 protein may bind to IGBP1-AS1 DNA. b RIP assay to identify the binding of IGBP1-AS1 to ZIC3. ${ }^{*} P$ value $<0.01$. c Upper Panel: Dual luciferase reporter assay to identify fluorescence intensity in cells with co-transfected truncated IGBP1-AS1 recombinant luciferase reporter vector and ZIC3 expression vector. ${ }^{*} P$ value $<0.01$; Lower Panel: dual luciferase reporter assay to identify fluorescence intensity of the cells with co-transfected mutated IGBP1-AS1 recombinant luciferase reporter vector and ZIC3 expression vector. ${ }^{*} P$ value $<0.01$. d ChIP assay to identify the binding ability of ZIC3 to the IGBP1-AS1 DNA binding site. ${ }^{* *} P$ value $<0.01$. e Schematic representation of the regulation of the IGBP1-AS1/miR-24-1/ZIC3 loop in BC. IGBP1-AS1 up-regulates the level of ZIC3 via suppressing miR-24-1. On the other hand, the elevated ZIC3 synchronously promotes the expression of IGBP1-AS1 by binding to its DNA at promoter region, further inhibiting BC cell proliferation and invasion

regulatory mechanisms could help us better understand the pathogenesis of $\mathrm{BC}$, which could the main direction of future research.

\section{Abbreviations}

ncRNAs: Noncoding RNAs; ATCC: American Type Culture Collection; FBS: Fetal bovine serum; RT-qPCR: Real-time quantitative polymerase chain reaction; ISH: In situ hybridization; DMSO: Dimethyl sulfoxide; RIP: RNA immunoprecipitation; AGO2: Argonaute 2; IVIS: Interactive Video Information System.

\section{Acknowledgements}

Special thanks for Dr. He Chen (Department of Pathology, The Second Affiliated Hospital of Jiaxing University, China), who provides samples and clinical information for this study. All authors collaborated in the collection and interpretation of the data and contributed to the manuscript.

\section{Authors' contributions}

DQC wrote the manuscript, YFF prepared the figures, FW edited the manuscript. All authors read and approved the final manuscript.

\section{Funding}

This work was supported by the Zhejiang Province Basic Public Welfare Research Project (GF18H160036).

\section{Availability of data and materials}

The datasets used and analyzed during the current study are available from the corresponding author on reasonable request.

\section{Ethics approval and consent to participate}

Written informed consent for the biological studies was obtained from each patient involved in the study, and the study was approved by the Ethics Committee of The Affiliated Woman Hospital of Zhejiang University. All animal studies were approved by the Animal Experimental Committee of The Affiliated Woman Hospital of Zhejiang University

\section{Consent for publication}

Written consent for publication was obtained from all the patients involved in our study. No identifiable individual patient or health person was involved in this study.

\section{Competing interests}

The authors declare that they have no competing interests.

Received: 22 August 2019 Accepted: 16 April 2020

Published online: 07 May 2020

\section{References}

1. Chen W, et al. Cancer statistics in China, 2015. CA Cancer J Clin. 2016:66:115-32. https://doi.org/10.3322/caac.21338.

2. Davis SL, Eckhardt SG, Messersmith WA, Jimeno A. The development of regorafenib and its current and potential future role in cancer therapy. Drugs Today. 2013;49:105-15. https://doi.org/10.1358/ dot.2013.49.2.1930525.

3. Tempero MA. Multidisciplinary management of pancreatic cancer. J Natl Compr Cancer Netw. 2015;13:700-2. 
4. Narayan M, et al. Trastuzumab-induced HER reprogramming in "resistant" breast carcinoma cells. Cancer Res. 2009:69:2191-4. https://doi. org/10.1158/0008-5472.CAN-08-1056.

5. Yarmishyn AA, Kurochkin IV. Long noncoding RNAs: a potential novel class of cancer biomarkers. Front Genet. 2015;6:145. https://doi. org/10.3389/fgene.2015.00145.

6. Ponting $\mathrm{CP}$, Oliver PL, Reik W. Evolution and functions of long noncoding RNAs. Cell. 2009;136:629-41. https://doi.org/10.1016/j. cell.2009.02.006

7. Jalali S, Kapoor S, Sivadas A, Bhartiya D, Scaria V. Computational approaches towards understanding human long non-coding RNA biology. Bioinformatics. 2015;31:2241-51. https://doi.org/10.1093/bioinforma tics/btv148.

8. Vikram R, Ramachandran R, Abdul KS. Functional significance of long non-coding RNAs in breast cancer. Breast Cancer. 2014;21:515-21. https ://doi.org/10.1007/s12282-014-0554-y.

9. Zhang $H$, et al. Up-regulation of miR-24-1-5p is involved in the chemoprevention of colorectal cancer by black raspberry anthocyanins. Br J Nutr. 2019;122:518-26. https://doi.org/10.1017/S0007114518003136.

10. Zhang W, et al. LINC01088 inhibits tumorigenesis of ovarian epithelial cells by targeting miR-24-1-5p. Sci Rep. 2018;8:2876. https://doi. org/10.1038/s41598-018-21164-9.

11. Braoudaki M, et al. miR-15a and miR-24-1 as putative prognostic microRNA signatures for pediatric pilocytic astrocytomas and ependymomas. Tumour Biol. 2016;37:9887-97. https://doi.org/10.1007/s1327 7-016-4903-7.

12. Ma G, Dai W, Sang A, Yang X, Li Q. Roles of ZIC family genes in human gastric cancer. Int J Mol Med. 2016;38:259-66. https://doi.org/10.3892/ ijmm.2016.2587.

13. Yang $B$, et al. MiR-564 functions as a tumor suppressor in human lung cancer by targeting ZIC3. Biochem Biophys Res Commun. 2015;467:6906. https://doi.org/10.1016/j.bbrc.2015.10.082.
14. Koblizek M, Lebedeva A, Fiser K. FlowlO: flow cytometry standard conformance testing, editing, and export tool. Cytometry Part A J Int Soc Anal Cytol. 2018. https://doi.org/10.1002/cyto.a.23563.

15. Dong J, et al. HMGA2-FOXL2 axis regulates metastases and epithelial-tomesenchymal transition of chemoresistant gastric cancer. Clin Cancer Res. 2017;23:3461-73. https://doi.org/10.1158/1078-0432.CCR-16-2180.

16. Ding W, Ren J, Ren H, Wang D. Long noncoding RNA HOTAIR modulates MiR-206-mediated Bcl-w signaling to facilitate cell proliferation in breast cancer. Sci Rep. 2017;7:17261. https://doi.org/10.1038/s41598-017-17492 $-x$.

17. Gokmen-Polar Y, Vladislav IT, Neelamraju Y, Janga SC, Badve S. Prognostic impact of HOTAIR expression is restricted to ER-negative breast cancers. Sci Rep. 2015;5:8765. https://doi.org/10.1038/srep08765.

18. Milevskiy MJ, et al. Long-range regulators of the IncRNA HOTAIR enhance its prognostic potential in breast cancer. Hum Mol Genet. 2016;25:326983. https://doi.org/10.1093/hmg/ddw177.

19. Li W, et al. Downregulation of LncRNA GAS5 causes trastuzumab resistance in breast cancer. Oncotarget. 2016;7:27778-86. https://doi. org/10.18632/oncotarget.8413.

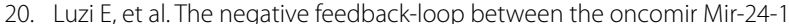
and menin modulates the Men 1 tumorigenesis by mimicking the "Knudson's second hit". PLoS ONE. 2012;7:e39767. https://doi.org/10.1371/journ al.pone.0039767.

\section{Publisher's Note}

Springer Nature remains neutral with regard to jurisdictional claims in published maps and institutional affiliations.
Ready to submit your research? Choose BMC and benefit from:

- fast, convenient online submission

- thorough peer review by experienced researchers in your field

- rapid publication on acceptance

- support for research data, including large and complex data types

- gold Open Access which fosters wider collaboration and increased citations

- maximum visibility for your research: over $100 \mathrm{M}$ website views per year

At BMC, research is always in progress.

Learn more biomedcentral.com/submissions 